\title{
ON THE COMPLEXITY OF THE ONE-TERMINAL NETWORK DESIGN PROBLEM
}

\author{
Nimrod MEGIDDO \\ Department of Statistics, Faculty of Social Sciences and Mathematical Sciences, Tel Aviv University, Ramat-Aviv, Tel Aviv, Israel
}

Received January 1982

Revised March 1982

\begin{abstract}
The one-terminal network design problem considered here is to select a subset of the set of potential edges so as to minimize the sum of construction cost plus expected usage cost with discounting. We distinguish between easy and hard cases of this problem.
\end{abstract}

Network design, telpak, spanning tree, NP-hard

We will consider one-terminal network design problems of the sort of Section A2.1 in Garey and Johnson's book [2]. We will explore the borderline between easy and hard problems of this kind (from the computational complexity point of view). A related problem is the capacitated spanning tree problem (denoted ND5 in [2]). Another related paper is [3] where a linear programming line of attack is adopted. The reader is referred to Section A2.1 in [2] for additional references.

The general problem is to select a subset $E^{\prime} \subset E$ of edges from a graph $G=(V, E)$ so that the total cost associated with the subgraph $G^{\prime}=\left(V, E^{\prime}\right)$ is minimized. The total cost consists of the cost of constructing the roads corresponding to $E^{\prime}$ plus the expected cost of traveling within the subnetwork $G^{\prime}$. The latter may be discounted over time. We will show that simple subcases of this problem are NP-hard. Thus, the restrictive assumptions that we list below strengthen in fact the NP-hardness results. First, we note that the multi-terminal problem is NP-hard even if all the demands are equal; this follows from the fact that the problem of finding the shortest total path length spanning tree is NP-hard [2, p. 206].

We consider only one-terminal problems, i.e. the demand for traveling is between each vertex $v$ and one distinguished vertex $t$ (the terminal). We assume all demands are equal, i.e., for example, at each vertex $v$ there lives one user who has to commute daily between $v$ and $t$. Two extreme cases are easy. First, if the cost of commuting is zero, i.e. only construction cost should be minimized, then our problem is that of the minimum spanning tree. Second, if the cost of construction is zero then the total length of paths from all $v$ 's to $t$ has to be minimized and this is achieved by the tree of shortest routes from $t$ to all $v$ 's. This is because each user travels the shortest distance possible whatsoever. This tree is found very efficiently by Dijstra's shortest-path algorithm (see [1]). If demands are not all equal then minimizing the cost of construction alone if NP-hard even when all demands are either zero or one, since this is in fact the Steiner tree problem in graphs (ND12). The one-terminal problem of minimizing the total weighted path length is solved, of course, by the tree of shortest routes from $t$ to all $v$ 's.

We now turn to the case where the two different costs are positive. We further assume that the costs are proportional to the length $d(i, j)$ of the edge $(i, j)$. We first claim that the optimal solution can be assumed, without loss of generality, to be a tree. This is argued as follows. Let $x_{i j}$ denote the number of users who will travel, on their way to $t$, along the edge $(i, j)$ in the direction from $i$ to $j$. Since exactly one user travels from $i$ to $t$ (not necessarily along the edge $(i, t)), \Sigma_{j}\left(x_{i j}-x_{j i}\right)=1$. Let $a$ denote the cost of constructing a unit-length of road and let $b$ denote the unit travel cost ( $a$, $b>0$ ). Thus, the total cost associated with the edge $(i, j)$ is zero if $x_{i j}=0$ and equals $\left(a+b \cdot x_{i j}\right)$ $\cdot d(i, j)$ if $x_{i j}>0$. We have to minimize the sum of all edge-costs subject to $\Sigma_{j}\left(x_{i j}-x_{j i}\right)=1, x_{i j} \geqslant 0$. 
Since the objective function is concave, there is always a basic optimal solution. However, all basic solutions of this problem correspond to spanning trees.

An obvious consequence of the tree argument is that the case of equal lengths is easy. For construction cost is equal for all spanning trees and hence the tree of shortest routes is again the optimal solution.

Our main result is the following.

Theorem. For all $a, b>0$, the one-terminal network design problem with equal demands and exactly two different edge-lengths is NP-hard.

Proof. We will show that 3-satisfiability reduces to our problem. Let $E_{j}=x_{j} \vee y_{j} \vee z_{j}, j=1, \ldots, m$, be the clauses, where $x_{j}, y_{j}, z_{j}$ are from the set $\left\{v_{1}\right.$, $\left.\bar{v}_{1}, \ldots, v_{p}, \bar{v}_{p}\right\}$. Construct the following network. Associate vertices with each $E_{j}, v_{i}$ and $\bar{v}_{i}$ and let $t$ be an additional vertex. Let there be edges of the forms $\left(v_{i}, t\right),\left(\bar{v}_{i}, t\right)$ and $\left(v_{i}, \bar{v}_{i}\right) i=1, \ldots, p$, and also let there be edges of the form $\left(E_{j}, x_{j}\right),\left(E_{j}, y_{j}\right)$ and $\left(E_{j}, z_{j}\right)$ for $j=1, \ldots, m$. Let the length of $\left(v_{i}\right.$, $\bar{v}_{i}$ ) be equal to $a$ and let the length of all other edges be $2 a+b$.

First, suppose that the formula $E_{1} \wedge \cdots \wedge E_{m}$ is satisfiable and let $s \subset\left\{v_{1}, \bar{v}_{1}, \ldots, v_{p}, \bar{v}_{p}\right\}$ be an appropriate assignment of truth-values, i.e. $S \cap E_{j}$ $\neq \varnothing, j=1, \ldots, m$, and $\left|S \cap\left\{v_{i}, \bar{v}_{i}\right\}\right|=1, i=1, \ldots, p$. Consider the following solution for the network design problem. All edges $\left(v_{i}, \bar{v}_{i}\right)$ are constructed and also $(w, t)$ is constructed if and only if $w$ is in $S$. Finally, for each $E_{j}$ construct one edge $\left(E_{j}, w\right)$ such that $w \in S \cap\left\{x_{j}, y_{j}, z_{j}\right\}$. Obviously, the total construction cost is equal to $a^{2}+a(p+m)(2 a+$ $b)$ which is also the cost of minimum spanning tree. The total travel cost is $b p(2 a+b)+b p(3 a+$ $b)+b m \cdot 2(2 a+b)$.

On the other hand, in any optimal solution exactly one of the two edges $\left(v_{i}, t\right),\left(\bar{v}_{i}, t\right)$ will be constructed. For if $\left(\bar{v}_{i}, t\right)$ exists then it is less expensive to construct $\left(v_{i}, \bar{v}_{i}\right)$ (at the cost of $a^{2}$ ) for $v_{i}$ so that $v_{i}$ travels via $\bar{v}_{i}$ (at the cost of $b(3 a+b))$ than to construct $\left(v_{i}, t\right)$ to let $v_{i}$ travel directly (at the total cost of $(a+b)(2 a+b))$. Also, each $E_{j}$ should have exactly one of the three edges incident upon $E_{j}$ (all of the same cost) constructed for him. Thus, in any optimal solution the construction cost is the cost of a minimum spanning tree, i.e., $a^{2} p+a(p+m)(2 a+b)$. Since each $E_{j}$

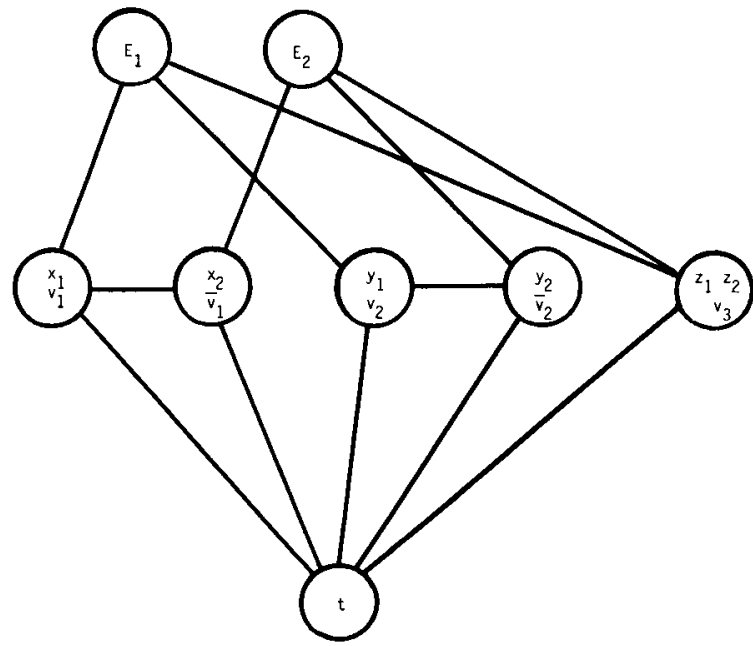

Fig. 1.

has to travel at least the distance of $2(2 a+b)$ to $t$ and each pair $v_{i}, \bar{v}_{i}$ always travels altogether $a+$ $2(2 a+b)$, it follows that a total traveling distance of $p \cdot(a+2(2 a+b))+m \cdot 2(2 a+b)$ is an optimal value which is feasible if and only if our formula is satisfiable. That enables each $E_{j}$ to travel no more than the minimum requires in any case (see Fig. 1). Note that precisely one member of the pair $v_{i}, \bar{v}_{i}$ is selected in this way.

It is only natural to expect, in view of the theorem that a locally optimal solution (i.e., a subtree which cannot be improved upon by just interchanging two edges) may not be globally optimal. This is illustrated in Fig. 2. It can be easily verified that with respect to $a=b=1$ the $(20,20$, $5)$ spanning tree is locally optimal but the $(19,19$, 5) spanning tree is the global minimum.

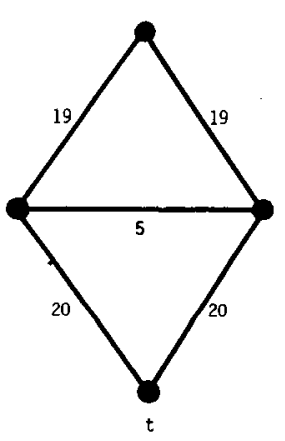

Fig. 2. 


\section{References}

[1] A.V. Aho, J.E. Hopcroft and J.D. Ullman, The Design and Analysis of Computer Algorithms, Addison-Wesley, Reading, MA (1974).
[2] M.R. Garey and D.S. Johnson, Computers and Intractability: A Guide to the Theory of NP-Completeness, Freeman, San Francisco (1979).

[3] B. Rothfarb and M. Goldstein, "The one-terminal telpak problem", Operations Res. 19, 156-169 (1971). 